ROUGH DRAFT-COMMENTS WELCOME

\title{
WHY DO WE NEED LABORATORY EXPERIMENTS IN POLITICAL SCIENCE?
}

\author{
Elinor Ostrom \\ Workshop in Political Theory and Policy Analysis \\ Department of Political Science \\ Indiana University \\ Center for the Study of Institutional Diversity \\ Arizona State University
}

(C) 2007 by author

Paper to be presented at the 2007 American Political Science Association annual meeting, Chicago, IL, August 30-September 2, 2007.

This paper draws in part on "The Value-Added of Laboratory Experiments for the Study of Institutions and Common-Pool Resources,” Journal of Economic Behavior \& Organization 61(2) (October 2006): 149-63. The author is appreciative of funding from the National Science Foundation, comments by James Walker, and the wonderful editing of Patty Lezotte.

\section{Workshop in Political Theory and Polic y Ana lysis}

\author{
Indiana University, 513 North Park, Bloomington, IN 47408-3895 USA
}

phone: 812.855.0441 / fax: 812.855.3150 / workshop@indiana.edu / http://www.india na.edu/ workshop 


\section{WHY DO WE NEED LABORATORY EXPERIMENTS IN POLITICAL SCIENCE? Elinor Ostrom}

\section{Introduction}

When I served the American Political Science Association as president during the 199697 academic year, I was asked to address most regional association meetings as is the convention of our linked associations. At each meeting, I focused on lessons learned about collective action from experimental research as well as from the field. Since the number of political scientists using experimental methods was very small at that time, some colleagues reacted negatively to this effort of mine to use every available opportunity to advocate this research method to a group of scholars who were quite skeptical about the value added of experimental techniques. I am very pleased to see the number of political scientists using experimental methods rising over time. I am delighted to be asked to present a paper on a panel of the 2007 APSA meetings devoted to experimental methods. This last ten years has seen a significant growth in the use of laboratory experiments in political science.

Given a reputation as an avid field researcher, colleagues still ask me why I "bother” with conducting experiments. They ask questions such as: "Why would you pay any attention to outcomes in an experiment." "They are such 'artificial worlds' and you have such rich data sets available to you." "What more can you possibly learn about institutions and resource governance from laboratory experiments that you have not already learned from empirical research conducted in field settings?”

My first response is that we always learn more from multiple research modes than we learn by relying on one method alone. Further, experimental research enables us to test the impact of specific variables in repeated controlled settings—something that is not available to 
scholars studying only field settings. To test theory adequately, we need to use methods that together combine external and internal validity. One gains external validity in doing field research, but internal validity in the laboratory. When political scientists use both methods related to one set of theoretical questions, advances in our understanding are multiplied.

In this paper, I would like to provide a brief overview of what we have learned from experimental research about common-pool resources about the impact of specific types of variables learned. Fieldwork has taught us that many biophysical, economic, political, and cultural factors affect the likelihood of resource users to overcome perverse incentives for organizing themselves, to establish boundaries, to craft rules for sustainable harvesting, to monitor their own rules, and to sanction colleagues who break these rules (Agrawal, 2001). In the field, we do not observe universal success in overcoming the multiple dilemmas that resource uses faced. In many systems, however, users have behaved contrary to the theoretical predictions derived in most resource economics textbooks of the 1980s and 1990s that managing common-pool resources requires either government imposed management or the division of a resource into private property. ${ }^{1}$

When conducting field research, one of the frustrating aspects is that so many variables are involved that one is never certain that one has isolated the specific variable—or limited set of variables - that causes an outcome. A good way to understand which components of a commonpool resource situation affect behavior and outcomes, and how, is to study a simplified version in an experimental laboratory. In the laboratory, the researcher carefully establishes the specific

\footnotetext{
${ }^{1}$ Our experiments have been stimulated by extensive field research on the impact of institutions on the performance of irrigation systems (Ostrom, 1992; Tang, 1992; Lam, 1998; Shivakoti and Ostrom, 2002), inshore fisheries (Schlager, 1994; Acheson, 2003; Acheson and Gardner, 2004; Yandle and Dewees, 2003), pastoral groups (Agrawal, 1999; Kaul, 1996), and forest governance (Gibson, McKean, and Ostrom, 2000; Varughese and Ostrom, 2001; Poteete and Ostrom, 2004; Gibson, Williams, and Ostrom, 2005; Moran and Ostrom, 2005; Hayes and Ostrom, 2005; Ostrom and Nagendra, 2006).
} 
components of the theoretical situation to be studied and controls other variables so they do not confound the analysis.

Thus, in this paper, I will provide a general overview of the experimental studies of behavior and outcomes in common-pool resource dilemmas conducted by Roy Gardner, James Walker, and myself, as well as other colleagues at Indiana University, or by colleagues at other universities who have replicated key experimental designs. I will focus primarily on the lessons we have learned from these experiments as a core part of a larger research program combining theoretical developments, field research, and laboratory experiments of relevance to political scientists. $^{2}$

In Section 2, common-pool resources and appropriation dilemmas will be defined.

Section 3 describes the sparse design we developed for our baseline appropriation experiment to include just the core aspects that underlie appropriation dilemmas in field settings: a non-linear payoff function based on the decisions of more than two appropriators who obtained no information about other subjects' actions. The subjects were homogeneous in their endowments and were not allowed to communicate, to keep the baseline as simple as possible. The average payoffs achieved in the baseline experiments did approach the Nash equilibrium of the noncooperative game, but with some unexplained pulsing behavior which was replicated by agentbased models created by Deadman (1997) and by Jager and Janssen (2002).

In Section 4, experiments that introduced face-to-face communication are described. In these experiments, subjects were authorized to communicate with one another in a group setting before returning to their terminals to make their own private decisions. Introducing an opportunity for “cheap talk,” where agreements are not enforced by an external authority, is

\footnotetext{
${ }^{2}$ For details of the specific designs and data obtained, readers a re encouraged to refer to the published articles referred to herein.
} 
viewed within the context of non-cooperative game theory as irrelevant. The same outcome is predicted as in the baseline experiment, but the experimental findings are dramatically different. An initial set of communication experiments retained the homogeneity of asset structure but did examine the difference between allocating subjects a small asset endowment versus a larger endowment. The last part of this section provides an overview of the experiments exploring whether communication would help to overcome the problems of heterogeneity of asset structure.

Section 5 provides an overview of the series of experiments where we changed the payoff component to allow subjects to sanction one another at a cost to themselves. Since using this option produces a benefit for all at a cost to the individual, the game-theoretic prediction is that no one will choose the costly sanctioning option. We had observed frequent instances of users sanctioning one another in the field and wanted to explore whether this would occur in a carefully designed environment controlling for other potentially confounding variables.

Section 6 synthesizes the experiments where we changed the authority rule to allow subjects to covenant with one another to determine their investment levels and to adopt a sanctioning system if they wished. Again, the predicted outcome is the same since the subjects now faced a multi-level dilemma and had to invest their own resources to enforce their decisions. In all three of these structurally changed appropriation experiments, however, subjects demonstrate their willingness and ability to search out and adopt better outcomes than those predicted. Since many political scientists have bought the argument made by Garrett Hardin (1968) regarding the helplessness of individuals caught in a "Tragedy of the Commons," the findings from these experiments (combined with extensive field research) should challenge that argument and establish that not all users of a common-pool resource are trapped in endless 
tragedy. We can go on in our research to ascertain the factors that affect the likelihood of overcoming common-pool resource tragedies rather than just recommending that national governments impose new rules.

\section{The Definition of a Common-Pool Resource}

A common-pool resource, such as a lake or ocean, an irrigation system, a fishing ground, a forest, the internet, or the stratosphere, is a natural or man-made resource from which it is difficult to exclude or limit users once the resource is provided by nature or produced by humans (Ostrom, Gardner, and Walker, 1994). One person’s consumption of resource units, such as water, fish, or trees, removes those units from what is available to others. Thus, the trees or fish harvested by one user are no longer available for others. The difficulty of excluding beneficiaries is a characteristic that is shared with public goods, while the subtractability of the resource units is shared with private goods.

When the resource units produced by a common-pool resource have a high value and institutional constraints do not restrict the way resource units are appropriated (an open access situation), individuals face strong incentives to appropriate more and more resource units leading eventually to congestion, overuse, and even the destruction of the resource itself. If some individuals reduce their appropriation levels, the benefits are shared with others whether they cut their harvesting or not. Some individuals may free ride on the costly actions of others unless ways are found to reduce free riding as an attractive strategy.

Consequently, the joint users of a common-pool resource face an “appropriation problem” given the incentive for individuals to appropriate more resource units when acting independently than they would if they could find some way of coordinating their appropriation activities. Joint users of a common-pool resource often face many other problems including 
assignment problems, technological externality problems, provision problems, and maintenance problems (Ostrom, Gardner, and Walker, 1994). The specific character of each of these problems differs substantially from one resource to the next. In this review, I focus on appropriation problems, since they are what most readers associate with the well known "tragedy of the commons" (Hardin, 1968).

\section{The Baseline Appropriation Experimental Design}

In our initial effort to design a baseline experiment, we wanted to start with a static, experiment that was as simple as we could specify it without losing crucial aspects of the problems that appropriators face in the field. To approximate some of the complexity of field settings (as well as the classic textbook example of a common-pool resource), however, we wanted to examine behavior in an appropriation situation with a non-linear transformation function based on Gordon (1954) and Scott (1955). We also wanted to include a sufficient number of players that knowledge of outcomes did not automatically provide information about each player's actions-again similar to the field. In this survey, I can only briefly discuss the series of experiments and a broad description of the changes in results obtained. All procedures and specifications are thoroughly documented in books and article cited in this article.

The first question we wished to address with a baseline experiment was whether subjects' behavior in this stark dilemma situation would be similar to that the Nash equilibrium predicted by non-cooperative game theory. The experiments were conducted at Indiana University by recruiting students from the general undergraduate population and indicating that they would be making decisions in an "economic choice situation" where they earn funds depending on their own and others decisions. 
Subjects were assigned an equal number of tokens in each round and told they could invest in two markets. The first market yielded a fixed rate of return for each token invested. The second market (the CPR) yielded a rate of return dependent upon the total number of tokens invested by all subjects in an experiment. The payoffs assigned to tokens invested in the second market were a concave function, $F$, which depended on the number of tokens, $x_{i}$, allocated to this market. Initially, if subjects invested small quantities of tokens, the sum of the subjects' actions, $\sum x_{i}$, generated better outcomes than the safe fixed rate of return. On the other hand, if the subjects allocated a sufficiently large number of their tokens, the outcome they receive was less than their best alternative. In other words, allocating too many assets to the common-pool resource would be counterproductive. Specifically the payoff to a subject was given by:

$$
\begin{array}{ll}
\text { we } & \text { if } x_{i}=0 \\
w\left(e-x_{i}\right)+\left(x_{i} / \sum x_{i}\right) F\left(\sum x_{i}\right) & \text { if } x_{i}>0 .
\end{array}
$$

If subjects put all of their tokens into Market 1 , they received a certain return equal to the amount of their endowment times an unchanging rate, $w$. If subjects put some of their endowed assets into the outside option, Market 1, and the rest in Market 2 (the CPR) they received part of their return from the outside option and the rest from their proportional investment in the commonpool resources times the total output of the common-pool resource as determined by function $F$.

\subsection{Predicted Outcomes for a Common-Pool Resource in the Laboratory}

In the baseline experiments, we utilized the following equation for the transformation function, $F$, measured in units of output (outcome units).

$$
23\left(\sum \mathrm{x}_{\mathrm{i}}\right)-25\left(\sum \mathrm{x}_{\mathrm{i}}\right)^{2}
$$


In the first set of experiments, we allocated 10 tokens to each subject in each round. In later experiments, we allocated 25 tokens. Their outside opportunity was valued at \$.05 per token. They earned \$.01 on each outcome unit they received from investing tokens in the common-pool resource. Subjects were informed that they would participate in an experiment that would last no more than two hours. The number of rounds in each experiment varied between 20 and 30 rounds. In addition to being told the payoff function specifically, subjects were provided with look-up tables that eased their task of determining outcomes depending on their own and others' decisions.

With these specifications, the predicted outcome for a finitely repeated game where subjects are not discounting the future and each subject is assumed to be maximizing monetary returns, is for each subject to invest 8 tokens in the common-pool resource for a total of 64 tokens. By design, the prediction is the same for both endowment levels. At this level of investment, they would each earn $\$ .66$ per round in the 10-token experiments and $\$ .70$ per round in the 25-token experiments (players were paid one-half of their computer returns in the 25-token experiments to keep the payoffs roughly similar). The players could, however, earn considerably more if the total number of tokens invested was 36 tokens (rather than 64 tokens) in the common-pool resource. This optimal level of investment would earn each subject $\$ .91$ per round in the 10-token experiment and $\$ .83$ per round in the 25-token experiment.

\subsection{Behavior in a Sparse Experimental N-Person, Repeated Appropriate Dilemmas}

Subjects interacting in baseline experiments substantially overinvested as predicted. Subjects in the 10-token experiments achieved, on average, 37 percent of the maximum earning from the common-pool experiment available to them while subjects in the 25-token experiments received -3 percent (Ostrom, Gardner, and Walker, 1994: 116). However, at the individual level, 
subjects rarely invested 8 tokens, which is the predicted level of investment at equilibrium. Instead, all experiments provided evidence of an unpredicted and strong pulsing pattern in which individuals appear to increase their investments in the common-pool resource until there is a strong reduction in yield, at which time they tend to reduce their investments leading to an increase in yields. The pattern is repeated over time. At an aggregate level, behavior approximates the predicted Nash equilibrium in the 10-token experiments, but is far lower than predicted in the early rounds of the 25-token experiment and only begins to approach the predicted level in later rounds. No game-theoretical explanation yet exists for the pulsing pattern or the substantial difference between the 10-token and the 25-token experiments. Those who study the impact of harvest technology in the field, however, will feel quite comfortable with this result. Large-scale trawlers and mechanized forest harvesting equipment are frequently thought to generate much less sustainable patterns than smaller boats or hand saws.

In response to post-experiment questions, subjects explained that they were using several heuristics. One of the heuristics they used was to invest more in the common-pool resource whenever the rate of return on the previous round was above $\$ .05$ (what they could earn in their next best alternative) and less if the return was below $\$ .05$. With such heuristic strategies, equilibrium is never reached at the individual level. Each player is revising his or her response to the results obtained in the most recent round creating considerable turbulence in the outcomes jointly reached.

\subsection{Replication in Agent-Based Models}

An extremely interesting follow-up study was undertaken by Peter Deadman (1997) in which artificial agents were programmed to use a variety of heuristics similar to those used by the human subjects in these experiments and to interact in a simulated environment that exactly 
replicated the baseline experiments. Deadman found that the specific results obtained in any series of runs depended on the particular heuristic (or mix of heuristics) programmed, but the artificial agents did consistently produce the same kind of pulsing returns and the consistent difference between 10-token and 25-token environments were also observed. Deadman describes his results:

As in CPR experiments, the group performance for the simulation follows an oscillating pattern in which high performance leads to over investment in the CPR and the resultant drop in performance causes a reduction in group-wide investment in the CPR. ... Still more interesting is the observation that the simulations perform similarly to subjects in laboratory experiments in terms of average performance over time. At the ten token endowment, the simulations perform near the Nash equilibria over time. At the 25 token endowment, the simulations perform near zero percent of optimum over time. (Deadman 1997: 175-76)

Jager and Janssen (2002) also developed a multi-agent model using the consumat framework derived from social psychology (Jager, Janssen, and Vlek, 2002). They thought that they could replicate the data from the baseline CPR experiments described above with an assumption that individual subjects differed in regard to their Social Value Orientations (SVOs). In their first series of simulations, they were indeed able, as Deadman had done independently, to explain the aggregated pattern of appropriation behavior. When we made the individual subject data available to them, Jager and Janssen were not as successful in replicating individual appropriation decisions as they had been in simulating aggregate outcomes. Jager and Janssen (2002) did suggest that the cognitive processes that subjects use appear to be important in behavior. Those with low aspiration levels may too soon lock into a habitual response. Further, those agents "conforming to the Homo psychologius have a better performance than the Homo economicus in approximating the empirical data” (p. 98). 


\section{Structural Changes in the Laboratory}

The findings from the baseline experiments were broadly consistent with predictions from non-cooperative game theory and not consistent with the findings from the field that many groups found ways of achieving cooperation among appropriators. The key challenge that we faced was which component of the base-line experiment to change in our effort to try to identify what factors in the field were more likely than others to affect positively levels of cooperation. One of the most obvious factors in relatively small groups of appropriators is the capabilities of these groups to engage in face-to-face communication.

\subsection{Face-to-Face Communication among Homogeneous Subjects}

In the repeated communication experiments, subjects first made ten rounds of decisions in the context of the baseline appropriation game. After the tenth round, subjects listened to an announcement that told them they would have an open group discussion before each of the next rounds of the experiment. The subjects left their terminals and sat in a circle facing one another. After each discussion, they returned to their terminals to enter their anonymous decisions. With the permission of the subjects, the discussions were tape recorded and then transcribed verbatim after the full completion of the experiments.

After each decision round, they learned what their aggregate investments had been, but not the decisions of individual players as in the baseline experiments. Thus, they learned whether total investments were greater than those they had agreed to. While in many rounds, subjects did exactly as they had promised one another they would do, some defections did occur. If promises were not kept, subjects used this information about the aggregate investment levels to castigate the unknown participant who had not kept to their agreement. 
This opportunity for repeated face-to-face communication was extremely successful in increasing joint returns in the 10-token experiments where subjects obtained close to 100 percent of the maximum available returns. There were only 19 instances out of 368 total opportunities where a subject invested more in the common-pool resource than agreed upon for a 5 percent defection rate (Ostrom, Gardner, and Walker, 1994: 154). In the 25-token experiments, subjects also improved their overall performance. The temptation to defect, however, was greater in the 25-token experiments. Our conclusion in completing an analysis of these experiments was:

Communication discussions went well beyond discovering what investments would generate maximum yields. A striking aspect of the discussion rounds was how rapidly subjects, who had not had an opportunity to establish a well-defined community with strong internal norms, were able to devise their own agreements and verbal punishments for those who broke those agreements. ... In many cases, statements like 'some scumbucket is investing more than we agreed upon' were a sufficient reproach to change defectors’ behavior. (Ostrom, Gardner, and Walker, 1994: 160)

That subjects had internalized norms regarding the importance of keeping promises is evidenced by several of their behaviors. Calling on others to join them in cutting back on their investments in the common-pool resource led most subjects to change their investment pattern. Secondly, subjects were indignant about evidence of investment levels higher than that promised and expressed their anger openly. Third, those who broke their promise tended to revert to the promised level after hearing the verbal tongue-lashing of their colleagues. The findings from these initial communication experiments are consistent with a large number of studies of the impact of face-to-face communication on the capacity of subjects to solve a variety of social dilemma problems (see Ostrom and Walker, 1991; Sally, 1995).

\subsection{Content Analysis of Communications}

Adam Simon and David Schwab (2006) have conducted an in-depth analysis of the verbatim transcripts to assess the potential relevance of Social Identity Theory (Turner, 1987; 
Hogg and Tindale, 2003) which predicts that individuals place themselves in identifiable roles as a particular role increases in salience. In this theoretical approach, when individuals see themselves as a member of a potential group, they may use solidarity language to increase the likelihood of other participants to seek group benefits rather than seeking their own personal, short-term benefits. The transcripts were coded independently by three coders who categorized phrases into multiple categories. In their analysis, Simon and Schwab correlated the number of solidarity words used and the number of defections (individual investment made above the level the group had agreed upon) observed for each period of an experiment. They found that defections were inversely correlated to the number of solidarity words used. When combined with a measure of early closure (an agreement reach in an early round leading to reduced time spent in each further communication round), they found that "each solidarity word is associated with a highly statistically significant $(p<.01)$ average drop of .06 in total defections” (p. 15 of W06-37). By drawing on the literature of Social Identity Theory, Simon and Schwab help to explain the strong results of these communication experiments that traditional game theory had not been able to explain. This analysis is very relevant for political scientists interested in the role of informal political communication (see Huckfeldt and Sprague, 1991; Kenny, 1998).

\subsection{Replications of Face-to-Face Communication among Homogeneous Subjects}

One of the great advantages of laboratory experiments is that they can be replicated as well as modified by other researchers so that one can gain ever greater confidence in the findings. One of the first replications of our CPR experiments was conducted by Rocco and Warglien (1995), who found very similar outcomes in the baseline, no-communication situation as well as in a replication of the face-to-face communication setting. They were interested, in addition, to the question of whether similar results could be obtained in a setting where 
communication was not organized on a direct face-to-face basis, but rather via computerized exchange of information. Thus, they used identical structural variables as we had earlier for their own face-to-face design and then the same structural variables for a third design where they limited communication to a form of computerized exchange. The effect of communication, when not conducted on a face-to-face basis, was greatly reduced in contrast to their own face-toface experiments.

Another very interesting series of replications and extensions was conducted by JuanCamilo Cardenas (2000) using field laboratories set up in school buildings in rural Colombia rather than a computer-based laboratory on a university campus. Cardenas initially invited over 200 villagers to participate in a series of common-pool resource experiments—several closely paralleled the ones conducted at Indiana University and discussed above and others that extended the questions that could be addressed. The villagers that Cardenas invited were actual users of local forests for the extraction of firewood, natural fibres, and log timber as well as local water resources. One of the basic questions he wanted to pursue was whether experienced villagers who were heavily dependent on local forests for wood products would behave in a manner broadly consistent with that of undergraduate students at an American Big 10 University.

The answer to this first question turned out to be positive. He wrote his instructions in Spanish and in a manner that would be easily understood by villagers. Instead of tokens—which are an easy medium for undergraduates — he asked villagers to decide on how many months a year they would spend in the forest gathering wood products as contrasted to using their time otherwise. Each villager had a copy of a payoff table, which was the same as the other seven participants. It showed that as the number of months that any individual would spend in the 
forest, that individual would gain more returns. The return to all of them, however, depended on all of them keeping their harvesting time to a very low level.

In the baseline, no-communication experiments, Cardenas found a similar pattern as we had found with subjects from Indiana University. Villagers substantially overinvested in the resource. While there was considerable variation among groups, villagers on average achieved 57.7 percent of their optimal return in the last three rounds of the baseline experiments (Cardenas, 2000: 316). The daily wage that most of the villagers could earn at the time of the experiment was around 7,000 pesos (or around U.S. $\$ 5.40$ at the time). If they all invested at an optimal level they would earn around 12,900 pesos. They did earn around 7,884 pesos in these experiments for the two or three hours they were involved in initial practice sessions and the actual experiments themselves.

Face-to-face communication enabled them to increase efficiency on average to 76.1 percent of optimal. Considerable variation among groups existed, which Cardenas was able to explain using information about the participants filled in after that experiment was completed. He found, for example, that when most members of the group were already familiar with common-pool resources such as the collective use of a mangrove, they used the communication rounds more effectively than when most members of the group were dependent primarily on their own assets. Cardenas also found that "social distance and group inequality based on the economic wealth of the people in the group seemed to constrain the effectiveness of communication for this same sample of groups” (Cardenas, 2000: 317; see also Cardenas, 2003).

\subsection{Face-to-Face Communications among Heterogeneous Subjects}

Steven Hackett, Edella Schlager, and James Walker (1994) conducted a series of CPR experiments where they explored whether communication could ameliorate the problems 
identified in field settings related to heterogeneity among appropriators (R. Hardin, 1982;

Johnson and Libecap, 1982; Libecap and Wiggins, 1984; Isaac and Walker, 1988; Wiggins and Libecap, 1987; Hackett, 1992).

The task of agreeing to and sustaining agreements for efficient CPR appropriation is more difficult for heterogeneous appropriators because of the distributional conflict associated with alternative sharing rules. In heterogeneous settings, all appropriators may be made better off by adopting a new rule, some will benefit more than others, depending upon the sharing rule chosen. Consequently, appropriators may fail to cooperate on the adoption of a sharing rule because they cannot agree upon what would constitute a fair distribution of benefits produced by cooperating.

In order to address appropriator heterogeneity, the Hackett, Schlager, and Walker experimental design allows for two levels of input endowments. One subset of appropriators has large endowments of tokens (24); the other appropriators have small token endowments (8). Group allocations to the CPR at the asymmetric Nash equilibrium are greater than optimal, but not all rents from the CPR are dissipated.

In order for communication to enhance joint payoffs to a heterogeneous set of subjects, they must agree on (1) the target level of group allocations to the CPR, (2) a rule for allocating the target input allocation across appropriators, and create (3) the necessary "social capital” to attenuate cheating, since agreements are nonbinding. The existence of heterogeneity in endowments and in historic allocation levels has no effect on the first problem, but is likely to elicit disagreement over second problems. Disagreements impair social capital.

Subjects knew with certainty the total number of decision makers in the group, their own token endowment and that of the others, the total number of tokens in the group, the 
transformation function, and the number of decision rounds in the current treatment condition. After each round, subjects were shown a display that recorded their payoffs in each market for that round, total group token allocations to the CPR, and a total of their cumulative profits for the experiment, but not the allocations made by specific other players.

Subjects participated in two (consecutive) ten-round sequences of the asymmetric game. In the first ten rounds, subjects were not allowed to communicate, but face-to-face communication was allowed during the second set of ten interactions. Prior to each ten-round treatment sequence, four subjects were assigned the "large" token endowment, while the other four subjects were assigned the "small" endowment. Two different mechanisms were used for assigning the large and small endowment positions: random and auction (based on Güth, 1988, 1992). In the first method, large endowments were assigned randomly prior to the ten decision rounds without communication, and again prior to the ten decision rounds with communication. A multiple-unit ascending price auction was used as the alternative mechanism for assigning endowment positions because of its demand-revealing characteristics. In particular, the price paid for the large endowment position should theoretically correspond with the maximum value placed on this position by the subject with the fourth highest valuation.

The opportunity to communicate led to a noticeable change in the pattern of allocations to the CPR. With the allocation rules agreed upon in communication rounds, subjects concentrated allocations to the CPR near the optimal allocation of 56 tokens in total. In the randomassignment and communication condition, individual CPR allocations of eight tokens represented the modal response (67\%). In the auction and communication condition, however, Hackett, Schlager, and Walker (1994) observed a spread of allocations clustered between six to ten tokens. For both treatment conditions with no-communication, they observe a level of rent 
accrual relatively close to that predicted by the Nash equilibrium (48.9\%). Thus, even in an environment of extreme heterogeneity in subject endowments, communication remains a powerful mechanism for promoting coordination, resulting in rents very close to those observed in the homogeneous decision setting discussed above.

\subsection{Face-to-Face Communication among a Subset of Subjects}

Three additional follow-up experimental designs were conducted by Pamela Schmitt, Kurtis Swope, and James Walker (2000). They used the same baseline design as first described with three variations in regard to the communication component of the situation. In all of their protocols for the communication aspects of the experiment (rounds 11 through 25), six out of the eight players were invited to communicate with one another in one location. In their first protocol, two of the remaining "players” were computerized decision makers whose decisions were each the result of a random draw of a number between 4 and 12 . The other six who could communicate with one another were informed about the constraints on the random draw for “Players 7 and 8.” In their second protocol, two of the players were real subjects that had been separated from the other six players. The real subjects did not face a constraint on their decisions. In the third protocol, the two separated players were constrained to invest between 4 and 12 tokens in the common-pool resource. As in the baseline experiments, subjects were informed about the aggregate investment of all eight players after each round.

In all three protocols, subjects substantially improved their overall efficiency in the communication rounds as contrasted to the non-communication rounds. Constraining who could communicate to six out of the eight players did, however, make a difference. The six communicating subjects were never certain if the aggregated investment level reflected higher investments by the two "non-communicating" players or whether some of the communicating 
groups did not follow an agreement. This uncertainty affected the capacity of the communicating group to make its own agreements in the first place in that they could always blame the outsiders for any major overinvestment.

There were also major differences in the behavior across the three protocols. The "outsiders" in Protocol 2 were the least constrained in their decisions. The members of the communicating group had the most difficulty in reaching agreements and following them in this second protocol. The six communicating subjects in Protocol 2 had a "scapegoat" they could blame for high investment levels in rounds 11 through 25. The subjects in Protocol 2 were less likely to come to an agreement in the first place, had a much higher deviation rate and size of deviation when they did agree, and obtained lower payoffs than in the earlier Ostrom, Gardner, and Walker experiments or in Protocols 1 or 3 . The problem of imperfect monitoring was less severe in Protocols 1 and 3. Some subjects among the communicating group were able to deviate without raising suspicion of cheating in these protocols, but they made only small increases over what the group had promised each other to do.

What this series of experiments found does have considerable implications for those trying to achieve an agreement in the field to limit harvesting from a common-pool resource. The results provide evidence that "communication is less likely to be effective in preventing over-harvesting in common-pool resource (CPR) environments in which a subset of appropriators either cannot or will not participate in collective action” (Schmitt, Swope, and Walker, 2000: 852). The lack of commitment by an outside group is not only a source of additional investment but also gives "insiders” a scapegoat to blame if their own harvests are higher than agreed upon. "The problem becomes more severe when outsiders have less 
constraints on their overall appropriation behavior and their ability to behave strategically” (ibid.).

\section{Sanctioning Experiments}

Participants in many field settings related to natural resources are able to communicate with one another on a face-to-face basis, at least from time to time, either in formally constituted meetings or at social gatherings. In most field settings, however, participants have also devised a variety of formal or informal ways of monitoring and sanctioning one another if rules are broken. In fact, the amazing speed with which irrigators responded to a perceived infraction while I was studying an irrigation system in Nepal led me to urge Roy Gardner and James Walker to help develop a game-theoretical model and experimental design to study monitoring and sanctioning in the lab. Engaging in costly monitoring and sanctioning behavior, even though often posed as a way of providing selective incentives, is not consistent with the theory of norm-free, complete rationality (Elster, 1989: 40-41). Thus, it was important to ascertain whether subjects in a controlled setting would actually make a costly decision in order to assess a financial punishment on the behavior of other participants as observed in the field. The short answer to this question is yes.

\subsection{The First Set of Sanctioning Experiments}

All sanctioning experiments used the 25-token design since appropriations had been much higher in this design (Ostrom, Walker, and Gardner, 1992). Subjects played ten rounds of the baseline game modified so that the individual contributions in each round were reported as well as the total outcomes. Subjects were then told that in the subsequent rounds they would have an opportunity to pay a fee in order to impose a fine on the payoffs received by another player. The fees ranged in diverse experiments from \$.05 to \$.20 and the fines from \$.10 to \$.80. 
In brief, the finding from this series of experiments is that much more sanctioning occurs than the zero level predicted.

Subjects react both to the intense cost of sanctioning and to the fee-to-fine relationships. Subjects sanction more when the cost of sanctioning is less and when the ratio of the fine to the fee is higher. Sanctioning is primarily directed at those who invested more in the CPR, but a few sanctions appear to be directed by those who had been fined in a form of "blind revenge" against those whose investments were lower than others and were thus suspected of having sanctioned them. In this set of experiments, subjects were able to increase their returns from the CPR modestly to 39 percent of maximum, but when the costs of fees and fines were subtracted from the total, these gains are wiped out. When subjects were given a single opportunity to communicate prior to the implementation of sanctioning capabilities, they were able to gain an average of 85 percent of the maximum payoffs ( 69 percent when the costs of the fees and fines were subtracted) (see Ostrom, Walker, and Gardner, 1992; Ostrom, Gardner, and Walker, 1994).

\subsection{Replications of Sanctioning Experiments}

Common-pool resource experiments have also been replicated and extended upon by Marco Casari and Charles Plott (2003). Casari and Plott wanted to explore whether an institution, which had been used in the Italian Alps for centuries and was thought to be highly effective, would generate positive results in a laboratory setting. The Alpine system had a relatively simple structure.

The population of a village developed a contract among themselves, subject to the approval of the regional government, called 'Carte di Regola', where they described a system for monitoring and sanctioning those who are discovered violating or exceeding patterns of use that the villagers agreed upon in the contract. The 'Carte di Regola' specified in advance the conditions under which a sanction could be inflicted on a person found in violation of the contract and the amount of the fine. ... Any villager could report a violation but he usually incurred a cost in the form of a monitoring effort to discover the violator and additional costs to bring him to court. A share of such a fine 
usually went to the person who discovered the violator in order to give an incentive to monitor. (ibid.: 218)

Casari and Plott used the same functional form for a payoff function as we had earlier used with Indiana University subjects (see equation 1), but they increased the monetary incentives by more than three-fold. ${ }^{3}$ Using Cal-Tech subjects, they first ran a baseline experiment that closely paralleled our earlier baseline experiments. Without communication or sanctioning, they found — as we had earlier found — that the resource was substantially overused, even more than the Nash equilibrium. Subjects earned only 28.4 percent of the optimal return, while the Nash equilibrium would have earned them 39.5 percent. They also found substantial variations among individual subjects in the amount they overused the resource, as we had earlier.

Casari and Plott then changed the transformation function and the payoffs of the game. They used two sanctioning conditions - weak and strong. In both conditions, after the decision regarding harvesting has been made and the total investment levels have been made public, a subject can select an option to inspect the decision made by any of the other subjects at a set cost. After this decision has been made, the harvesting decision of the inspected subject is made public information, but not the identity or number of subjects requesting an inspection. A fine is imposed for each unit appropriated above the announced level and transferred to the inspector. The "inspector" makes a profit when the fee that has been paid to carry out the inspection is less than the amount transferred, which is in turn dependent upon the amount that the inspected appropriator has exceeded the announced level. The weak sanctioning option did not change the predicted Nash equilibrium for the game, but the strong sanctioning option makes the predicted Nash equilibrium approach the socially optimal level of appropriation.

\footnotetext{
${ }^{3}$ Casari and Plott also changed the instructions given to players so as to make the differences between the three conditions very distinct. In general, the broad pattern of behavior in the base condition was very similar to our own earlier experiments.
} 
In the experiments conducted with weak sanctions, slightly over half of the actions were inspected—a higher level than predicted by classical game theory. And, subjects obtained closer to optimal levels of returns than they had without sanctions. In the strong sanction condition, the efficiency of the joint return was 94 percent, but when inspection fees are subtracted, the net return is 76.9 percent of optimal (ibid.: 238). Almost all actions were inspected, and it turned out that the lowest users were the more aggressive inspectors than the highest users.

Casari and Plott found that the subjects behaved in a manner consistent with having heterogeneous preferences rather than all having the same preferences monotonically aligned with the payoff available. Some individuals appear to be more spiteful than others. This helps to explain the success of the "Carte di Regola" system, as it would appear that it was able to use the "heterogeneity of preferences to socially advantageous ends" (ibid.: 241) by channeling the behavior of the more spiteful individuals into socially useful purposes. Overall, they found that the experiment mimicking the set of rules used in the Italian Alps greatly improved the efficiency of resource use as contrasted to the baseline experiment without the sanctioning options.

Casari and Plott provide a cogent and important theoretical explanation for their findings based on a proposed model of individual choice that they call a "heterogeneous, linear otherregarding model.” Their model predicts the outcomes of this experiment quite well, while the traditional model of individual behavior used in classical game theory does not explain the behavior in their common-pool resource experiments (or that of our own earlier experiments) well at all (except in the baseline condition). Given that the results from both types of experimental situations—as well as many other extensively replicated experimental situations— 
are not consistent with what is predicted from classical game theory, it is time to discuss the deep problem of animating institutional analysis.

\section{Covenanting Experiments}

In self-organized field settings, participants rarely impose sanctions on one another that have been devised exogenously as we did in the above sanctioning experiments. Sanctions are much more likely to emerge from an endogenous process of crafting their own rules, including the punishments that should be imposed if these rules are broken. Spending time and effort in a linked collective-choice situation designing rules creates a public good for all of those involved. Crafting rules for an operational situation is thus a second-level dilemma that theorists have argued is no more likely to be solved than the original commons dilemma. Non-cooperative game theory predicts that participants will not undertake such efforts. This is the foundation for the repeated recommendation that rules must be imposed on participants by external authorities who then assume responsibility for monitoring and enforcing these rules. Since self-organized rules are found in many local common-pool resource situations, it does appear that participants frequently do design their own rules contrary to the theoretical prediction. Few scholars are able to witness these processes, however, in the field.

In order to observe what happens in these settings, subjects who had already experienced baseline and sanctioning experiments were recalled and given an opportunity to make a collective choice in the laboratory to decide whether or not they would like to adopt a sanctioning mechanism, how much the fines and fees should be, and on the joint investment strategy that they would like to adopt. All of these groups were endowed with 25 tokens in each round. Four out of six experimental groups adopted a covenant in which they specified the 
number of tokens they would invest and the level of fines to be imposed. The fines determined by the participants ranged in size from $\$ .10$ to $\$ 1.00$ (Ostrom, Walker, and Gardner, 1992).

The groups that crafted their own agreements were able to achieve an average of 93 percent of the maximum in the periods after their agreement. And, the defection rate for these experiments was only 4 percent. The two groups that did not agree to their own covenant did not fare as well. They averaged 56 percent of the maximum available returns and faced a defection rate of 42 percent (ibid.). Consequently, those subjects that used an opportunity to covenant with one another to agree on a joint strategy, choose their own level of fines, received very close to optimal results based entirely on their own promises and their own willingness to monitor and sanction one another when it was occasionally necessary (see Frohlich, Oppenheimer, and Eavey, 1987, for similar findings).

\section{Imposed Rules}

Cardenas, Stranlund, and Willis (2000) report on another fascinating extension. In five experiments, the villagers were given a chance to communicate after the initial ten rounds of the baseline condition. In five other experiments, they were told that a new regulation would go into force that mandated that they should spend no more than the optimal level of time in the forest each round (which in this case was one month per villager). They were told that there would be a 50 percent chance that conformance to the rule would be monitored each round. The experimenter rolled a die in front of the subjects each round to determine whether an inspection would take place. If an even number showed up, there would be an inspection. The experimenter then drew a number from chits (numbered between 1 and 8) placed in a hat to determine who would be inspected. Thus, the probability that anyone would be inspected was 1/16 per round — a low but realistic probability for monitoring forest harvesting in rural areas. 
The monitor checked the investment of the person whose time had come without revealing the result to others. If the person was over the limit imposed, a penalty was subtracted from the payoff to that person. No statement was made to others whether the appropriator was complying with regulations or not.

The subjects in this experimental condition actually increased their withdrawal levels in contrast to behavior when no rule at all was imposed, but the subjects could communicate on a face-to-face basis. What was remarkable about this experiment was that subjects, who were simply allowed to communicate with one another on a face-to-face basis, were able to achieve a higher joint return than the subjects who had an optimal but imperfectly enforced external rule imposed on them. As the authors conclude:

We have presented evidence that indicates that local environmental policies that are modestly enforced, but nevertheless are predicted by standard theory to be welfareimproving, may be ineffective. In fact, such a policy can do more harm than good, especially in comparison to allowing individuals collectively to confront local environmental dilemmas without intervention. We have also ... presented evidence that the fundamental reason for the poor performance of external control is that it crowded out group-regarding behavior in favor of greater self-interest. (Cardenas, Stranlund, and Willis, 2000: 1731)

\section{Conclusion}

Let me now return to the question regarding the value-added of experimental research for political scientists and for the overall research program on the impact of diverse institutions on behavior and outcomes of users of common-pool resources. First, I hope a reader now recognizes that political scientists can learn a great deal from experimental research. The research reported on in this paper illustrates that solving common-pool resource dilemmas is non-trivial, but feasible for those directly involved if they can communicate with one another and agree on future actions. Without communication of some sort, however, subjects in the laboratory behave in the aggregate relatively close to the predicted Nash equilibrium of the non- 
cooperative game. When they cannot change each others expectations, individual behave as short-term, payoff maximizers. When scholars ask me whether solutions are likely to be achieved relatively easily for problems involving large, amorphous groups that have significant problems of communicating or choosing those authorized to communicate for them, such as the over-use of ocean fisheries or global warming, I respond in the negative (Ostrom et al., 1999). These are far more challenging problems than those faced by users of smaller resources who have authority to make their own rules.

Second, we have shown that groups that can communicate and design their own appropriation and sanctioning systems achieve substantial improvements—at times very close to optimal results. It is rarely feasible to observe such processes in the field but the findings are very important in regard to the importance of discourse and deliberation in a self-governing setting.

Third, subjects who have rules imposed on them—even ones that specify an optimal appropriation level for their environment—begin to cheat on one another relatively rapidly. With external monitoring that approximates what is found in many rural areas, such groups achieve far less than their own "cheap talk" agreements. Fourth, the experiments have shown that face to face communication can also help participants overcome problems of heterogeneity and sub-group divisions.

Thus, at a more general level, our experiments, along with field research and theoretical efforts, lead us to posit that the crucial variables to enhance cooperation in regard to commonpool resources and other forms of collective action, are reciprocity, individual reputations, and trust (Ostrom, 1998). Repeated interactions where participants get to gain trust that others are trustworthy and engaged in reciprocal relationships are difficult to achieve but lead to high levels 
of performance. Field research shows that many rules adopted by local resource users (and sometimes denigrated by policy analysis) affect reciprocity, reputations, and trust. Large groups with the authority to make their own rules, tend to create nested decision-making units so that smaller units can engage in effective communication and decision-making about aspects of a smaller subsystem. There are, however, no single rules guaranteed to have a positive effect in all settings (Ostrom, 1990; 2005).

Unfortunately, some policy advisors have thought that involving the users of a resource in some kind of participatory activity is easy and a good way to overcome resistance to external programs designed to protect resources. This is not the lesson that I derive from these results. Calling resource users to a single meeting and asking them "to participate" while telling them about what a project will do, are just exogenous changes which are likely to crowd out positive endogenous processes (Frey, 1994). These efforts are unlikely to create a setting in which reciprocity can be achieved among individuals who have a reputation for being trustworthy and thus enhance the overall level of trust in a group. 


\section{$\underline{\text { References }}$}

Acheson, James M. 2003. Capturing the Commons: Devising Institutions to Manage the Maine Lobster Industry. Hanover, NH: University Press of New England.

Acheson, James M., and Roy Gardner. 2004. "Strategies, Conflict, and the Emergence of Territoriality: The Case of the Maine Lobster Industry.” American Anthropologist 6(2) (June): 296-307.

Acheson, James M., and Roy Gardner. 2005. "Spatial Strategies and Territoriality in the Maine Lobster Industry.” Rationality and Society 17(3): 309-41.

Agrawal, Arun. 1999. Greener Pastures: Politics, Markets, and Community among a Migrant Pastoral People. Durham, NC: Duke University Press.

Agrawal, Arun. 2001. “Common Property Institutions and Sustainable Governance of Resources.” World Development 29(10): 1649-72.

Bardhan, Pranab K., and Jeff Dayton-Johnson. 2002. "Unequal Irrigators: Heterogeneity and Commons Management in Large-Scale Multivariate Research.” In The Drama of the Commons, National Research Council, Committee on the Human Dimensions of Global Change, ed. Elinor Ostrom, Thomas Dietz, Nives Dolšak, Paul Stern, Susan Stonich, and Elke Weber, 87-112. Washington, D.C.: National Academy Press.

Barnard, Chester. 1938. The Functions of the Executive. Cambridge: Harvard University Press.

Cardenas, Juan-Camilo. 2000. “How Do Groups Solve Local Commons Dilemmas? Lessons from Experimental Economics in the Field.” Environment, Development and Sustainability 2:305-22.

. 2003. "Real Wealth and Experimental Cooperation: Evidence from Field Experiments." Journal of Development Economics 70:263-89.

Cardenas, Juan-Camilo, John K. Stranlund, and Cleve E. Willis. 2000. "Local Environmental Control and Institutional Crowding-Out.” World Development 28(10):1719-33.

Casari, Marco, and Charles R. Plott. 2003. "Decentralized Management of Common Property Resources: Experiments with a Centuries-Old Institution.” Journal of Economic Behavior and Organization 51:217-47.

Coleman, James. 1973. The Mathematics of Collective Action. Chicago: Aldine.

Deadman, Peter. 1997. Modeling Individual Behavior in Common Pool Resource Management Experiments with Autonomous Agents. Ph.D. diss., University of Arizona, Tucson.

Deci, Edward L. 1975. Intrinsic Motivation. New York: Plenum Press. 
Dietz, Thomas, Elinor Ostrom, and Paul Stern. 2003. “The Struggle to Govern the Commons.” Science 302(5652):1907-12.

Elster, Jon. 1989. The Cement of Society: A Study of Social Order. Cambridge: Cambridge University Press.

Frey, Bruno. 1997. Not Just for the Money: An Economic Theory of Personal Motivation. Cheltenham, England: Edward Elgar.

1994. "How Intrinsic Motivation is Crowded Out and In.” Rationality and Society 6:33452.

Frohlich, Norman, Joe A. Oppenheimer, and Cheryl Eavey. 1987. "Choices of Principles of Distributive Justice in Experimental Groups.” American Journal of Political Science 31(3):606-36.

Fudenberg, D., B. Holmström, and Paul Milgrom. 1990. "Short-term Contracts and Long-term Agency Relationship.” Journal of Economic Theory 51:1-31.

Gardner, Roy, Elinor Ostrom, and James Walker. 1990. "The Nature of Common-Pool Resource Problems.” Rationality and Society 2(3) (July): 335-58.

Gibson, Clark, Margaret McKean, and Elinor Ostrom, eds. 2000. People and Forests: Communities, Institutions, and Governance. Cambridge, MA: MIT Press.

Gordon, H. Scott. 1954. "The Economic Theory of a Common Property Resource: The Fishery.” Journal of Political Economy 62:124-42.

Grafton, R. Quentin. 2000. "Governance of the Commons: A Role for the State? Land Economics 76(4):504-17.

Güth, Werner. 1988. "On the Behavioral Approach to Distributive Justice-A Theoretical and Experimental Investigation.” In Applied Behavioral Economics, vol. 2, ed. S. Maital. New York: New York University Press.

. 1992. "Distributive Justice: A Behavioral Theory and Empirical Evidence.” Ekonomiska Forskningsinstitutet Research Paper 6461, Stockholm.

Hackett, Steven. 1992. "Heterogeneity and the Provision of Governance for Common-Pool Resources.” Journal of Theoretical Politics 4(3):325-42.

Hackett, Steven, Edella Schlager, and James Walker. 1994. "The Role of Communication in Resolving Commons Dilemmas: Experimental Evidence with Heterogeneous Appropriators.” Journal of Environmental Economics and Management 27:99-126. 
Hardin, Garrett. 1978. "Political Requirements for Preserving our Common Heritage.” In Wildlife and America, ed. H. P. Bokaw, 310-17. Washington, D.C.: Council on Environmental Quality.

Hardin, Russell. 1982. Collective Action. Baltimore, MD: Johns Hopkins University Press.

Hayes, Tanya, and Elinor Ostrom. 2005. "Conserving the World's Forests: Are Protected Areas the Only Way?” Indiana Law Review 38(3):595-617.

Huckfeldt, Robert, and John Sprague. 1991. "Discussant Effects on Vote Choice: Intimacy, Structure and Interdependence.” Journal of Politics 53 (February): 122-58.

Isaac, R. Mark, and James Walker. 1988. "Group Size Effects in Public Goods Provision: The Voluntary Contributions Mechanism.” Quarterly Journal of Economics 103:179-99.

Jager, Wander, and Marco Janssen. 2002. Using Artificial Agents to Understand Laboratory Experiments of Common Pool Resources with Real Agents.” In Complexity and Ecosystem Management, ed. Marco Janssen, 75-102. Cheltenham, UK: Edward Elgar.

Jager, Wander, Marco Janssen, and C.A.J. Vlek. 2002. “How Uncertainty Stimulates OverHarvesting in a Resource Dilemma: Three Process Explanations.” Journal of Environmental Psychology 22:247-63.

Johnson, Ronald N., and Gary D. Libecap. 1982. "Contracting Problems and Regulation: The Case of the Fishery.” American Economic Review 72(5) (December): 1005-23.

Kaul, Minoti Chakravarty. 1996. Common Lands and Customary Law: Institutional Change in North India over the Past Two Centuries. New Delhi: Oxford University Press.

Kenny, C. 1998. “The Behavioral Consequences of Political Discussion: Another Look at Discussant Effects on Vote Choice.” Journal of Politics 60 (February): 231-44.

Kiser, Larry L., and Elinor Ostrom. 1982. “The Three Worlds of Action: A Metatheoretical Synthesis of Institutional Approaches.” In Strategies of Political Inquiry, ed. Elinor Ostrom, 179-222. Beverly Hills, CA: Sage.

Kreps, David M., Paul Milgrom, John Roberts, and Robert Wilson. 1982. "Rational Cooperation in the Finitely Repeated Prisoners’ Dilemma.” Journal of Economic Theory 27:245-52.

Laffont, Jean-Jacques, and David Martimort. 2002. The Theory of Incentives: The PrincipalAgent Model. Princeton, NJ: Princeton University Press.

Lam, Wai Fung. 1998. Governing Irrigation Systems in Nepal: Institutions, Infrastructure, and Collective Action. Oakland, CA: ICS Press. 
Libecap, Gary D., and Steven N. Wiggins. 1984. "Contractual Responses to the Common Pool: Prorationing of Crude Oil Production.” American Economic Review 74:87-98.

McGinnis, Michael, ed. 1999. Polycentricity and Local Public Economies: Readings from the Workshop in Political Theory and Policy Analysis. Ann Arbor: University of Michigan Press.

Moran, Emilio, and Elinor Ostrom. 2005. Seeing the Forests and the Trees: Human-Environment Interactions in Forest Ecosystems. Cambridge, MA: MIT Press.

Oakerson, Ronald J. 1992. “Analyzing the Commons: A Framework.” In Making the Commons Work: Theory, Practice, and Policy, ed. Daniel W. Bromley, et al., 41-59. San Francisco, CA: ICS Press.

Orbell, John M., and Robyn M. Dawes. 1993. “Social Welfare, Cooperators’ Advantage, and the Option of Not Playing the Game.” American Sociological Review 58(6) (December): 787-800.

Ostrom, Elinor. 1990. Governing the Commons: The Evolution of Institutions for Collective Action. New York: Cambridge University Press.

. 1992. Crafting Institutions for Self-Governing Irrigation Systems. San Francisco, CA: ICS Press.

. 1998. "A Behavioral Approach to the Rational Choice Theory of Collective Action.” American Political Science Review 92(1):1-22.

. 2005. Understanding Institutional Diversity. Princeton, NJ: Princeton University Press.

Ostrom, Elinor, Joanna Burger, Christopher Field, Richard B. Norgaard, and David Policansky. 1999. "Revisiting the Commons: Local Lessons, Global Challenges.” Science 284(5412) (April 9): 278-82.

Ostrom, Elinor, and Roy Gardner. 1993. "Coping with Asymmetries in the Commons: SelfGoverning Irrigation Systems Can Work.” Journal of Economic Perspectives 7(4) (Fall): 93-112.

Ostrom, Elinor, Roy Gardner, and James Walker. 1994. Rules, Games, and Common-Pool Resources. Ann Arbor: University of Michigan Press.

Ostrom, Elinor, and Harini Nagendra. 2006. "Insights on Linking Forests, Trees, and People from the Air, on the Ground, and in the Laboratory.” PNAS 103(51):19224-31.

Ostrom, Elinor, and James Walker. 1991. "Communication in a Commons: Cooperation without External Enforcement.” In Laboratory Research in Political Economy, ed. Thomas R. Palfrey, 287-322. Ann Arbor: University of Michigan Press. 
Ostrom, Elinor, James Walker, and Roy Gardner. 1992. "Covenants with and without a Sword: Self-Governance is Possible.” American Political Science Review 86(2) (June): 404-17.

Plott, Charles R., and David P. Porter. 1996. "Market Architectures and Institutional Testbedding: An Experiment with Space Station Pricing Policies.” Journal of Economic Behavior and Organization 31(2):237-72.

Poteete, Amy, and Elinor Ostrom. 2004. "Heterogeneity, Group Size and Collective Action: The Role of Institutions in Forest Management.” Development and Change 35(3) (June): 43561.

Rocco, Elena, and Massimo Warglien. 1995. "Computer Mediated Communication and the Emergence of ‘Electronic Opportunism'.” Venice, Italy: University of Venice, Department of Economics, Laboratory of Experimental Economics.

Sally, David. 1995. “Conversation and Cooperation in Social Dilemmas: A Meta-Analysis of Experiments from 1958 to 1992.” Rationality and Society 7:58-92.

Schlager, Edella. 1994. "Fishers’ Institutional Responses to Common-Pool Resource Dilemmas.” In Rules, Games, and Common-Pool Resources, ed. Elinor Ostrom, Roy Gardner, and James Walker, 247-66. Ann Arbor: University of Michigan Press.

Schlager, Edella, and Elinor Ostrom. 1992. "Property-Rights Regimes and Natural Resources: A Conceptual Analysis.” Land Economics 68(3) (August): 249-62.

Schmitt, Pamela, Kurtis Swope, and James Walker. 2000. "Collective Action with Incomplete Commitment: Experimental Evidence.” Southern Economic Journal 66(4):829-54.

Scott, Anthony D. 1955. “The Fishery: The Objectives of Sole Ownership.” Journal of Political Economy 63:116-24.

Shivakoti, Ganesh P., and Elinor Ostrom, eds. 2002. Improving Irrigation Governance and Management in Nepal. Oakland, CA: ICS Press.

Simon, Adam, and David Schwab. 2006. "Say the Magic Word: Effective Communication in Social Dilemmas.” Working Paper. New Haven, CT: Yale University.

Smith, Vernon L. 1982. "Microeconomic Systems as an Experimental Science.” American Economic Review 72 (December): 923-55.

Tang, Shui Yan. 1992. Institutions and Collective Action: Self-Governance in Irrigation. San Francisco, CA: ICS Press. 
1994. "Institutions and Performance in Irrigation Systems.” In Rules, Games, and Common-Pool Resources, ed. Elinor Ostrom, Roy Gardner, and James Walker, 225-45. Ann Arbor: University of Michigan Press.

Turner, J. 1987. Rediscovering the Social Group: A Self-Categorization Theory. London: Basil Blackwell.

von Wright, Georg Henrik. 1966. “The Logic of Action: A Sketch.” In The Logic of Decision and Action, ed. Nicholas Rescher, 121-36. Pittsburgh, PA: University of Pittsburgh Press.

Wiggins, Steven N., and Gary D. Libecap. 1987. "Firm Heterogeneities and Cartelization Efforts in Domestic Crude Oil.” Journal of Law, Economics, and Organization 3:1-25.

Williamson, Oliver E. 1975. Markets and Hierarchies: Analysis and Antitrust Implications. New York: Free Press.

Yandle, Tracy. 2001. "Market-Based Natural Resource Management: An Institutional Analysis of Individual Tradable Quotas in New Zealand's Commercial Fisheries.” Ph.D. diss., Indiana University.

Yandle, Tracy, and Christopher Dewees. 2003. "Privatizing the Commons...Twelve Years Later: Fishers' Experiences with New Zealand's Market-Based Fisheries Management.” In The Commons in the New Millennium: Challenges and Adaptations, ed. Nives Dolšak and Elinor Ostrom, 101-27. Cambridge, MA: MIT Press. 